\title{
Liver biopsy is still needed in liver transplantation recipients: a single center experience
}

\author{
Eman Abdelsameea1*, Ayman Alsebaey ${ }^{1}$, Nermine Ehsan², Ahmed Elrefaey², Khalid Aboelela ${ }^{3}$, \\ El-Sayed Ibrahim ${ }^{1}$ and Maha Elsabaawy
}

\begin{abstract}
Background: Liver transplantation is a final treatment for decompensated liver disease.

Aim: Description of post-liver transplant histopathology. We enrolled 89 patients divided into two groups according to if they underwent on demand liver biopsy ( $n=34 ; 38.2 \%)$ or not $(n=55 ; 61.8 \%)$. Albumin-bilirubin (ALBI) score and model for end-stage liver disease (MELD) assessed the degree of liver dysfunction.

Results: Patient underwent liver biopsy (LB) was $44.65 \pm 8.46$ years old, mainly males (88.2\%) with average MELD of $8.74 \pm 4.71$. Most patients were positive pre-transplant for HCV $(91.2 \%)$ and $29.4 \%$ had hepatocellular carcinoma on top of hepatitis C. Patients underwent LB had worse liver dysfunction by ALBI score ( $-2.62 \pm 0.6$ vs. $-2.96 \pm 0.5 ; p$ $=0.014$ ) but comparable MELD. The time till first biopsy was $19.88 \pm 11.22$ (4-44) months. It was not different statistically with various histopathology $(p>0.05)$. Histopathology of first biopsy was viral chronic hepatitis (50\%), acute rejection (20.6\%), steatohepatitis (11.8\%), chronic rejection (5.9\%), chronic hepatitis (5.9\%), biliary obstruction (2.9\%), and cytomegalovirus hepatitis (2.9\%). Most patients were F1 (38.2\%) and A1 (35.3\%). The immunosuppressive drug regimen had no impact on the histopathology $(p>0.05)$. Patients with hepatitis $C$ pre-transplant had in a descending manner the following histological diagnosis $(p=0.001)$ : viral chronic hepatitis $16(51.6 \%)$, acute rejection 7 (22.6\%), steatohepatitis 4 (12.9\%), chronic rejection 2 (6.5\%), biliary obstruction 1 (3.2\%), and CMV hepatitis 1(3.2\%). Some patients required on demand second $(n=9)$ and third biopsied $(n=5)$ that were the same as the first biopsy or completely different.
\end{abstract}

Conclusion: Liver biopsy is a useful tool for diagnosis of liver transplantation complications.

Keywords: Liver, Biopsy, Liver transplantation, Rejection, Recurrent post-transplant

\section{Background}

Liver transplantation (LT) is a final treatment for end stage liver diseases. LT improves the quality of life and is a definitive therapy for metabolic liver diseases as Wilson's disease and hemochromatosis. Liver transplantation is associated with a lot of complications for example nonfunctioning graft, rejection, vascular

\footnotetext{
* Correspondence: dreman555@yahoo.co.uk

'Department of Hepatology and Gastroenterology, National Liver Institute,

Menoufia University, Shebeen El-Koom 32511, Egypt

Full list of author information is available at the end of the article
}

complications, biliary complications, infections, and viral recurrence [1].

The diagnosis of the various events or complications can be done noninvasively with laboratory and radiological investigations as with vascular and biliary complications [2]. Nonetheless, some complications can be diagnosed only with the aid of liver biopsy as acute cellular rejection, recurrent autoimmune hepatitis, and steatohepatitis $[3,4]$.

Liver biopsy is invasive technique for obtaining samples from the liver tissue for histopathology. In fact, it is the golden standard for diagnosis of liver fibrosis and 
inflammation [5]. Besides, it can detect other associated diseases as steatosis and sarcoidosis. It is useful also for follow up, assessing progression, or regression of the disease and efficacy of therapy. Although liver biopsy is a safe maneuver, yet it is associated with some disadvantages, some of them may be life dangerous as bleeding. The common complications range from pain, hematoma, organ injury, and fistulation. Another point that may affect the histopathology is the heterogeneous distribution of the disease within the liver, along with inter and intra-observer variation [6].

This study aimed to describe the diagnostic role of liver biopsy in the recipients of liver transplantation.

\section{Methods}

This is a retrospective study that was conducted at National Liver Institute Hospitals, Menoufia University after approval by the institutional review board. Data were taken from the medical records in the liver transplantation unit.

From 2003 to 2015, about 198 adult patients underwent living donor liver transplantation. Eighty-nine patients were included in the study as 99 patients died and 10 patients travelled abroad and their data was not available. The patients were divided into two groups according to if they underwent on demand liver biopsy $(n=34$; $38.2 \%)$ or not $(n=55 ; 61.8 \%)$.

All the patients received intraoperative methylprednisolone as $10 \mathrm{mg} / \mathrm{kg}$ during the anhepatic phase and after operation they were shifted to the intensive care unit (ICU) [7]. In the ICU, the following lab tests were done every $12 \mathrm{~h}$ : liver functions, renal functions, complete blood counts, international normalized ratio (INR), uric acid, electrolytes, C-reactive protein, cholesterol, serum phosphate, serum lactate, fibrinogen, and fibrin degradation products. Doppler on the hepatic artery, portal vein, and hepatic veins was done every $4 \mathrm{~h}$. Prophylactic antibiotics, antifungals, anticoagulants, and antiviral as acyclovir were given [1].

The patients were usually shifted to ward on the 8th day where anticoagulant therapies are shifted to antiplatelets as dipyridamole, and the investigations were done once daily. After discharge, the patients had a visit every week for 2 months then monthly till the end of the first year after which the visit was every 3 months.

In that era, the only available treatment for hepatitis $\mathrm{C}$ virus $(\mathrm{HCV})$ infection was pegylated interferon/ribavirin with poor results. The direct acting antivirals for hepatitis $\mathrm{C}$ were not available. The treatment was deferred after 6-12 months and was given if the fibrosis stage was F3-4 Metavir by liver biopsy with features of recurrent viral hepatitis. Lamivudine and hepatitis B immunoglobulin were given for patients with hepatitis B.
The following immunosuppressive drugs were used: calcineurin inhibitors (tacrolimus, cyclosporine), steroids, mTOR inhibitors (sirolimus, everolimus), mycophenolate mofetil, or mycophenolic acid. Induction with basiliximab was used for patients with pre-transplant renal impairment or calcineurin inhibitors sparing.

The usual applied immunosuppressive therapy was the triple steroids, tacrolimus, and mycophenolate mofetil per os. Patients intolerant to mycophenolate mofetil were shifted to mycophenolic acid per os. Patients developed side effects with tacrolimus were shifted to oral cyclosporine. Patients with renal impairment shifted to sirolimus and in the last years to everolimus [8,9].

On the time of biopsy, the following regimens were applied: $17.6 \%$ (cyclosporine and mycophenolate mofetil), $17.6 \%$ (sirolimus and mycophenolic acid), 17.6\% (tacrolimus and mycophenolate mofetil), $14.7 \%$ (cyclosporine, sirolimus, and mycophenolate mofetil), 14.7\% (tacrolimus monotherapy), 5.9\% (sirolimus monotherapy), 2.9\% (tacrolimus, everolimus, and mycophenolate mofetil), $2.9 \%$ (tacrolimus, sirolimus, and mycophenolate mofetil), 2.9\% (everolimus and mycophenolate mofetil), and 2.9\% (cyclosporine monotherapy).

Protocol biopsies were not adopted by our center. Only on demand biopsies were applied with the following conditions: sudden elevation or deterioration of the liver enzymes not responding to adjustment of the immunosuppressive drugs, confirmation of cytomegalovirus hepatitis, pre-pegylated interferon/ribavirin therapy, cholestasis of unclear diagnosis, unclear diagnosis of liver focal lesions, suspicion of recurrent disease, or steatohepatitis. It is to be noted that the vascular causes were excluded by Doppler ultrasonography and CT angiography. The biliary causes were excluded by preliminary ultrasonography confirmed by magnetic resonance cholangiopancreatography.

Sonar guided transcutaneous liver biopsy under complete aseptic technique was done [10]. The slides were read by 2 blinded pathologists to avoid interobserver variation. Fibrosis stage and the histological activity were assessed by Metavir score [10].

Calculations for assessing degree of liver dysfunction: Albumin-bilirubin (ALBI) score [11] $=\left(\log _{10}\right.$ bilirubin $\mu \mathrm{mol} / \mathrm{L} \times 0.66)+($ albumin $\mathrm{g} / \mathrm{L} \times-0.085)$.

Model for end-stage liver disease (MELD) [12] $=3: 78$ $\ln [$ serum total bilirubin $(\mathrm{mg} / \mathrm{dL})]+11: 2 \ln [\mathrm{INR}]+9: 57$ $\ln [$ serum creatinine $(\mathrm{mg} / \mathrm{dL})]+6: 43$.

\section{Statistical analysis}

Data were statistically analyzed using IBM $^{\oplus}$ SPSS $^{\bullet}$ Statistics $^{\circledR}$ version 21 for Windows (IBM Corporation, North Castle Drive, Armonk, NY, USA). Data were expressed as mean \pm standard deviation and number percentage for nominal data. All $p$ values are 2 tailed, with values < 
0.05 considered statistically significant. Comparisons between two groups were performed using the Student's $t$ test for normally distributed data, and Mann-Whitney test for nonparametric data that lacks the normal distribution. CHI-squared test $\left(\chi^{2}\right)$ was done for nominal data comparison.

\section{Results}

In the current study as shown in Tables 1 and 2, most patients who underwent liver biopsy $(n=34)$ were 44.65 \pm 8.46 years old and mainly males $30(88.2 \%)$. The age ranged from 25 to 58 years old. They had average $8.74 \pm$ 4.71 MELD score. The laboratory parameters were as follows: total bilirubin $1.96 \pm 2.50 \mathrm{mg} / \mathrm{dL}$, serum albumin $4.11 \pm 0.62 \mathrm{~g} / \mathrm{dL}$, aspartate aminotransferase (AST) $111.79 \pm 67.08 \mathrm{U} / \mathrm{L}$, alanine aminotransferase (ALT) $125.59 \pm 70.42 \mathrm{U} / \mathrm{L}$, alkaline phosphatase (ALP) $541.45 \pm$ $1185.65 \mathrm{U} / \mathrm{L}$, gamma glutamyl transpeptidase (GGT) $486.3 \pm 653.2 \mathrm{U} / \mathrm{L}$, serum creatinine $0.98 \pm 0.29 \mathrm{mg} / \mathrm{dL}$, WBCs $6 \pm 2.2710^{9} / \mathrm{L}$, platelets $167.82 \pm 83.3210^{9} / \mathrm{L}$, and INR was $1.05 \pm 0.22$.

Wide fluctuations were found in the liver tests where with AST that ranged 30-270 U/L, ALT ranged 20-303 U/L, GGT 11-3408 U/L, and finally marked fluctuation with ALP 81-7120 U/L.

The indications of liver transplantation were diverse. From virological point, most patients who underwent liver biopsy were mainly HCV positive: $31(91.2 \%)$ and one case $1(2.9 \%)$ had dual hepatitis B virus (HBV) and $\mathrm{HCV}$ coinfection. Two patients were virus free. In fact patients who underwent liver biopsy had multiple indications as follows: HCV; $21(61.8 \%)$, hepatocellular carcinoma (HCC) on top of HCV; $10(29.4 \%)$, HCC on top of dual $\mathrm{HBV}$ and $\mathrm{HCV}$ coinfection; $1(2.9 \%)$, primary sclerosing cholangitis (PSC); $1(2.9 \%)$ and autoimmune hepatitis (AIH); $1(2.9 \%)$.

Patients were followed up for $51.53 \pm 15.92$ (10.0-93 months). The average time from transplant operation to the first biopsy was $19.88 \pm 11.22$ ranging from $4-44$ months.

The histopathology revealed 7 diagnoses in a descending pattern as the following (Fig. 1): chronic viral hepatitis, 17 (50\%); acute rejection, 7 (20.6\%); steatohepatitis, 4. (11.8\%); chronic rejection, 2 (5.9\%), chronic hepatitis, 2 (5.9\%); biliary obstruction, 1 (2.9\%); and cytomegalovirus (CMV) infection, 1 (2.9\%).

Chronic viral hepatitis was defined histologically by portal tract mononuclear inflammatory infiltrate with lymphoid aggregate formation, interface hepatitis, parenchymal necroinflammation, and steatosis as shown in Fig. 1a. Portal tract fibrosis varied in different cases, ranged from F0-F4 according to METAVAIR scoring system. Acute cellular rejection (ACR) revealed a wide range of histological presentations. A portal tract triad
Table 1 Characteristics of the liver transplant recipients that were biopsied

\begin{tabular}{lll}
\hline & $\mathrm{M} \pm \mathrm{SD}$ & Range \\
\hline Age years & $44.65 \pm 8.46$ & $25-58$ \\
Time till biopsy months & $19.88 \pm 11.22$ & $4-44$ \\
Follow-up months & $51.53 \pm 15.92$ & $10.0-93$ \\
Total bilirubin $\mathrm{mg} / \mathrm{dL}$ & $1.96 \pm 2.50$ & $0.3-9.9$ \\
Serum albumin $\mathrm{g} / \mathrm{dL}$ & $4.11 \pm 0.62$ & $2.7-5.1$ \\
AST U/L & $111.79 \pm 67.08$ & $30-270$ \\
ALT U/L & $125.59 \pm 70.42$ & $20-303$ \\
ALP U/L & $541.45 \pm 1185.65$ & $81-7120$ \\
GGT U/L & $486.3 \pm 653.2$ & $11-3408$ \\
Urea mg/dL & $41.97 \pm 16.98$ & $20-105$ \\
Serum creatinine mg/dL & $0.98 \pm 0.29$ & $0.5-1.7$ \\
Serum sodium mmol/L & $138.44 \pm 4.04$ & $129-146$ \\
Serum potassium mmol/L & $4.31 \pm 0.46$ & $3.8-5.7$ \\
Hemoglobin $\mathrm{g} / \mathrm{dL}$ & $12.71 \pm 2.09$ & $7.7-17.6$ \\
WBCs $10^{9} / \mathrm{L}$ & $6 \pm 2.27$ & $2.3-14.2$ \\
Platelets $10^{9} / \mathrm{L}$ & $167.82 \pm 83.32$ & $59-398$ \\
INR & $1.05 \pm 0.22$ & $0.8-2.1$ \\
MELD & $8.74 \pm 4.71$ & $3-28$
\end{tabular}

Liver Fibrosis

$\begin{array}{ll}\text { None } & 16(47.1 \%) \\ \text { F1 } & 13(38.2 \%) \\ \text { F2 } & 3(8.8 \%) \\ \text { F3 } & 2(5.9 \%)\end{array}$

Liver Activity

None $\quad 16(47.1 \%)$

A1 $12(35.3 \%)$

A2 $5(14.7 \%)$

A3 $1(2.9 \%)$

Histopathology

Acute rejection $\quad 7(20.6 \%)$

Viral chronic hepatitis $\quad 17(50 \%)$

Steatohepatitis $\quad 4(11.8 \%)$

Biliary obstruction $\quad 1(2.9 \%)$

CMV infection $1(2.9 \%)$

Chronic rejection 2(5.9\%)

Chronic hepatitis 2 (5.9\%)

$M \pm S D$ mean \pm standard deviation, $A S T$ aspartate aminotransferase, $A L T$ alanine aminotransferase, $A L P$ alkaline phosphatase, GGT gamma-glutamyl transferase,

WBC white blood cell, INR international normalized ratio, MELD model for endstage liver disease, $C M V$ cytomegalovirus

of histopathological findings in the form of mixed inflammatory cellular infiltrate, venular endothelietis, and bile duct injury was a typical diagnosis for ACR (Fig. 1b). Other histopathological changes were also noticed 
Table 2 Comparison of liver transplant recipients who did and did not undergo liver biopsy

\begin{tabular}{|c|c|c|c|c|}
\hline & \multicolumn{2}{|c|}{ Recipients $(n=89)$} & & \multirow[t]{3}{*}{$p$} \\
\hline & Non-biopsied & Biopsied & & \\
\hline & $n=55(61.8 \%)$ & $n=34(38.2 \%)$ & & \\
\hline Age years & $46.96 \pm 8.74$ & $44.65 \pm 8.46$ & & 0.222 \\
\hline MELD & $8.82 \pm 2.86$ & $8.74 \pm 4.71$ & & $0.258 \#$ \\
\hline ALBI score & $-2.96 \pm 0.50$ & $-2.62 \pm 0.60$ & & $0.014 \#$ \\
\hline Follow-up months & $38.40 \pm 20.42$ & $51.53 \pm 15.92$ & & 0.001\# \\
\hline \multicolumn{5}{|l|}{ Sex } \\
\hline Male & $53(96.4 \%)$ & $30(88.2 \%)$ & $83(93.3 \%)$ & \multirow[t]{2}{*}{0.197} \\
\hline Female & $2(3.6 \%)$ & $4(11.8 \%)$ & $6(6.7 \%)$ & \\
\hline \multicolumn{5}{|l|}{ Indication of LT } \\
\hline $\mathrm{HCV}$ & $33(60 \%)$ & $21(61.8 \%)$ & $54(60.7 \%)$ & \multirow[t]{6}{*}{0.192} \\
\hline $\mathrm{HCV}+\mathrm{HCC}$ & $18(32.7 \%)$ & $10(29.4 \%)$ & $28(31.5 \%)$ & \\
\hline HBV & $4(7.3 \%)$ & 0 & $4(4.5 \%)$ & \\
\hline $\mathrm{HCV}+\mathrm{HBV}+\mathrm{HCC}$ & 0 & $1(2.9 \%)$ & $1(1.1 \%)$ & \\
\hline PSC & 0 & $1(2.9 \%)$ & $1(1.1 \%)$ & \\
\hline $\mathrm{AlH}$ & 0 & 1 (2.9\%) & $1(1.1 \%)$ & \\
\hline
\end{tabular}

MELD model for end-stage liver disease, $A L B I$ albumin-bilirubin, $H C V$ hepatitis $C$ virus, $H B V$ hepatitis $B$ virus, $H C C$ hepatocellular carcinoma, $P S C$ primary biliary cholangitis, $\mathrm{AlH}$ autoimmune hepatitis

\#Mann-Whitney $U$ test

in cases of ACR such as interface hepatitis and perivenular parenchymal collapse. Steatohepatitis was diagnosed histologically by the presence of steatosis in hepatocytes, ballooning, and lobular inflammation with neutrophil rich (Fig. 1c). Liver biopsy diagnosed as chronic rejection revealed missing major bile ducts in portal tracts and arteriopathy. Chronic hepatitis other than viral etiology exhibited portal tract inflammation with spill over the interface and lobular inflammation in the form of apoptotic bodies, spotty, and confluent necrosis. Liver biopsy diagnosed as biliary obstruction revealed portal tract edema, mixed acute and chronic inflammatory cellular infiltrate, and ductular proliferation with intraluminal bile plugs as shown in Fig. 1d.

About $52.9 \%$ of patients had variable degrees of liver fibrosis mainly F1, 13 (38.2\%),then F2, 3 (8.8\%) and at last F3, 2 (5.9\%). None of them had F4 fibrosis. As regards, the histological activity of most of the patients had A1, 12 (35.3\%) then A2, 5 (14.7\%) and few were A3, $1(2.9 \%)$.

There was no statistically significant difference of the time till the first biopsy among the different diagnosis groups $(p=0.737)$. The time for each one was as follows: acute rejection, $15.14 \pm 13.69$ (04-44 months); viral chronic hepatitis, $20.76 \pm 10.3$ (06-44 months); steatohepatitis, $25.25 \pm 14.17$ (10-44 months); biliary obstruction, 11 (11-11 months); CMV infection, 15 (15-15 months); chronic rejection, $18.5 \pm$ 14.85 (08-29 months); and chronic hepatitis, $26.5 \pm$ 4.95 (23-30 months).
Patients that did $(n=34)$ and did not $(n=55)$ undergo liver biopsy were matched $(p>0.05)$ for age, sex, MELD score, indication of liver transplantation, and virology. The follow up period was statistically higher in the biopsied patients $(51.53 \pm 15.92$ vs. $38.40 \pm 20.42$ months, $p$ $=0.001)$. The liver dysfunction reflected by the ALBI score (Fig. 2) was statistically higher in the biopsied group $(-2.62 \pm 0.6$ vs. $-2.96 \pm 0.5 ; p=0.014)$.

Figure 3 shows the pattern of the histopathology in relation to the indication of liver transplantation. Patients with $\mathrm{HCV}$ had in a descending manner the following ( $p$ $=0.001)$ : viral chronic hepatitis $16(51.6 \%)$, acute rejection 7 (22.6\%), steatohepatitis 4 (12.9\%), chronic rejection 2 (6.5\%), biliary obstruction 1 (3.2\%), and CMV hepatitis 1 (3.2\%). The patient with dual HBV and HCV had viral chronic hepatitis related to HCV. The two viral free patients had chronic hepatitis.

On sub analysis $(p=0.024)$ as in Table 2 , patients with HCV had viral hepatitis (47.6\%), patients with HCC on top of HCV had viral hepatitis (60\%), patient with dual HBV and HCV had viral hepatitis, PSC and AIH had viral hepatitis. There was no statistically significant impact $(p=0.81)$ of the applied immunosuppressive drug regimen on the histopathology as shown in Table 3 and Fig. 4.

Some patients required a second and third biopsy. In the second biopsy $(n=9)$ the histopathology was acute rejection; 3 (33.3\%), viral chronic hepatitis; 3 (33.3\%), chronic rejection; 2 (22.2\%) and chronic hepatitis; 1 $(11.1 \%)$. In the third biopsy $(n=5)$ the histopathology 

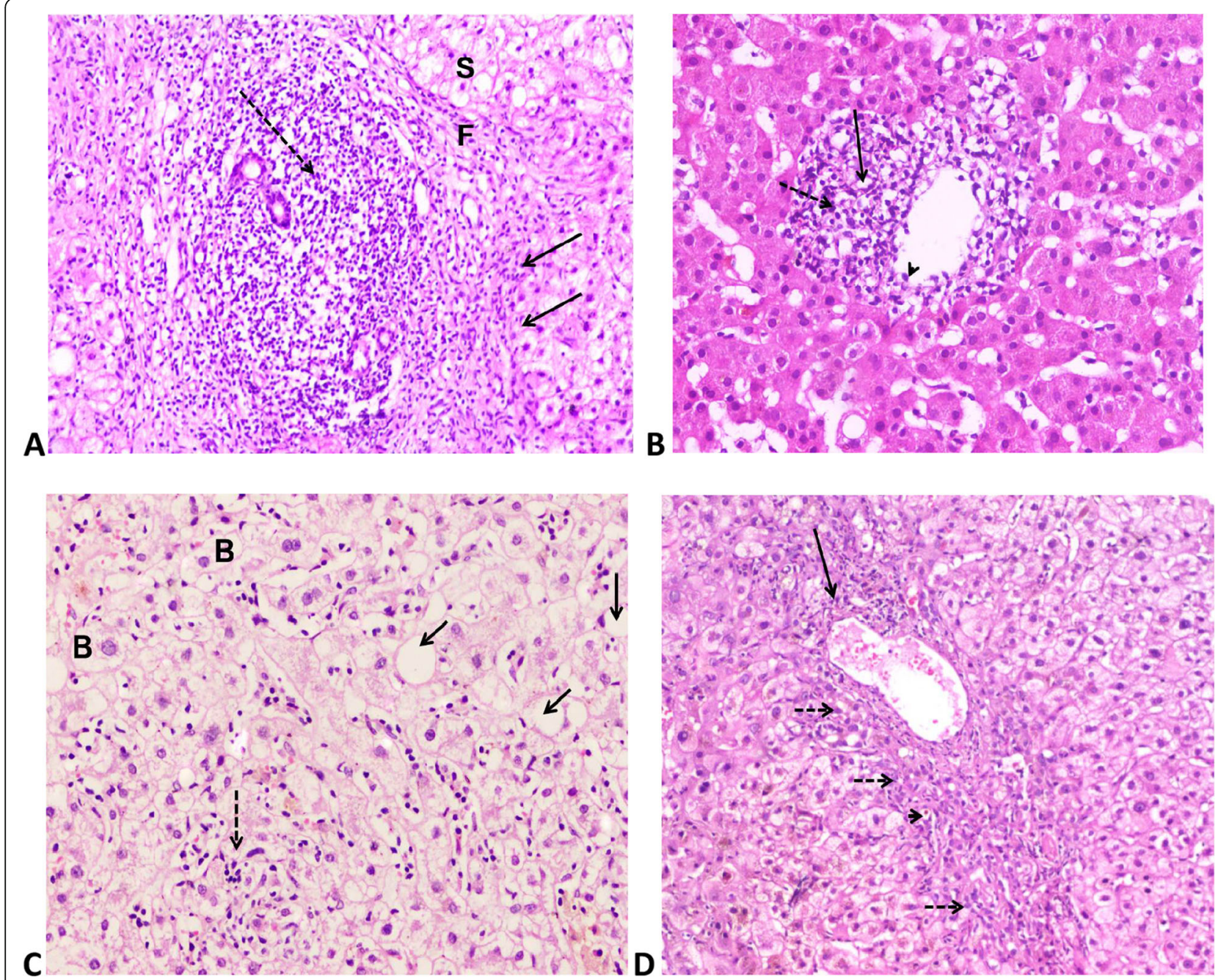

Fig. 1 Liver biopsy findings post liver transplantation: a Recurrent HCV infection showing portal tract mononuclear inflammatory infiltrate forming lymphoid aggregate (dashed arrow), interface hepatitis (arrow), fibrosis (F), and steatosis (S). b Acute cellular rejection demonstrating diagnostic portal tract triad of mixed cellular infiltrate (dashed arrow), bile duct injury (arrow), and venular endothelietis (arrow head). c Steatohepatitis exhibiting steatosis (arrows), ballooned hepatocytes (B), and neutrophilic rich infiltrate around hepatocytes (dashed arrow). d Biliary obstruction revealing mixed acute and chronic inflammatory cellular infiltrate (arrow), ductular proliferation (dashed arrow), and intraluminal bile plugs (arrow head)

was acute rejection, viral chronic hepatitis, biliary obstruction, CMV infection, and chronic rejection.

The findings of the second and the third biopsy may be the same as the first one or changing to another histopathology as in Table 4. One patient with acute rejection developed another attack and one proceeded to chronic rejection. Patients with viral hepatitis: some had the same diagnosis and some developed acute rejection, chronic rejection, biliary obstruction, and finally CMV hepatitis.

\section{Discussion}

Liver biopsy is the gold standard for diagnosis of many liver diseases and is a useful tool for follow-up. Despite the many advantages it has also drawbacks. It is an invasive maneuver that causes pain and liability for bleeding that may be life threatening. Organ perforation is one of the reported complications. Sometimes it may miss or underestimate the diagnosis as some diseases do not affect all parts of the liver evenly requiring both right and left lobe biopsy. Besides, there is inter-observer variation [13].

In the context of liver transplantation liver biopsy may be done early or late. The early indications are worsening or non-decreasing liver functions, re-rise of the liver functions after improvement and protocol biopsy. The late indications are rise of the liver enzymes from baseline, failed normalization after treated event, imaging abnormalities, and protocol biopsies [3]. 


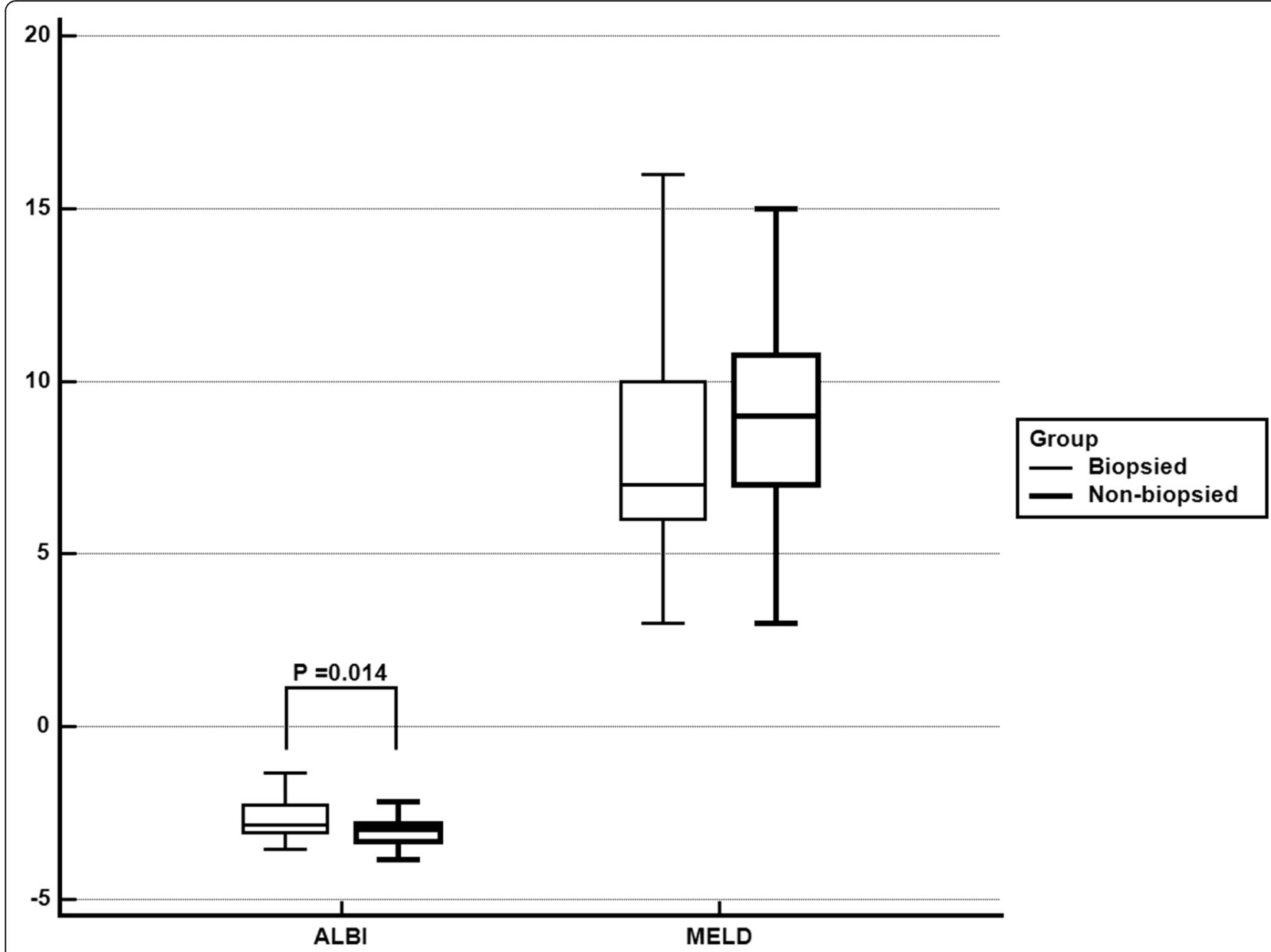

Fig. 2 Comparisons of the MELD and ALBI score in patients who did and did not undergo liver biopsy

The common histopathology depends on the time of the liver biopsy. Early, the diagnosis is usually small for size, acute cellular rejection, confirmation of vascular event, or severe recurrent of diseases as fibrosing cholestatic hepatitis. In the late one, it is common to find recurrent diseases as viral hepatitis, acute, or chronic rejection and sometimes recurrent malignancies [3, 4].

The need of liver biopsy till now is mandatory for diagnosis and assessment of some diseases as acute, chronic rejection, steatohepatitis, and de novo autoimmune hepatitis. Liver biopsy is needed for confirmation of uncertain laboratory or radiology diagnosis as with CMV or liver tumors. The enthusiasm of liver biopsy has faded away in many aspects and supplanted nowadays by noninvasive parameters as seen with recurrent viral hepatitis where laboratory and radiological parameters are sufficient to commence antiviral therapy.

On reviewing the studies on the utility of liver biopsy post-transplant; $\mathrm{Yu}$ et al. [14] analyzed 50 biopsies out of 27 patients (2002-2003). The time till biopsy ranged from $5 \mathrm{~h}$ to 330 days. The main histological diagnosis was acute rejection (48.2\%), preservation-reperfusion injury (25.9\%), chronic rejection (14.8\%), hepatic artery thrombosis (11.1\%), drug induced hepatitis (11.1\%), CMV (3.7\%), and recurrent hepatitis (3.7\%).

An Iranian study [15] analyzed 382 post LT biopsies from 287 patients. The average age was 28 (1-64 years). The main indication of liver transplantation was HBV (20.2\%) followed by AIH (17.7\%). The time till biopsy varied from few hours till 261 days post-transplant. Acute cellular rejection was the most common finding (47\%) followed by hepatic artery related ischemic changes. Some patients had normal liver histopathology $(17.7 \%)$.

In another study, Kanodia et al. [16] studied 57 biopsies from 35 patients (January 2010 to July 2014). Some patients underwent repeated biopsies. The average age was 53 years and most of them were males. The mean bilirubin was $5.54 \mathrm{mg} / \mathrm{dL}$, AST (197 U/L), ALT (298 U/ $\mathrm{L})$, and ALP (256 U/L). The most common indication for LT was alcoholic cirrhosis (25.71\%), cryptogenic (17.14\%), HBV related (17.14\%), and Wilson's disease 


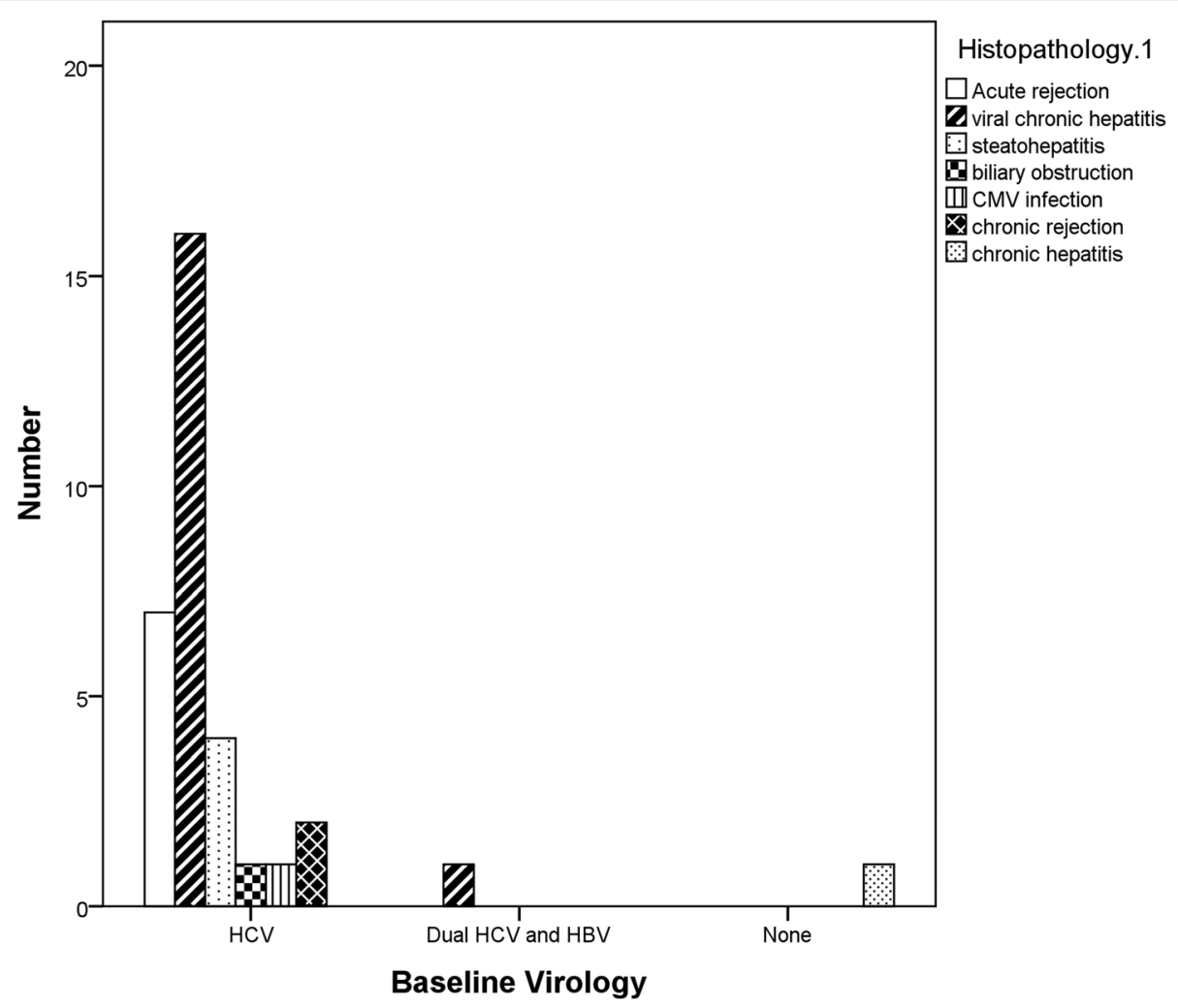

Fig. 3 Correlations between the indication of liver transplantation and the histopathology

(11.42\%). Few cases with $\mathrm{HCV}, \mathrm{AIH}$, and PSC were present. The time till biopsy ranged from 1 to 980 days. The most common histological diagnosis was acute rejection (55.36\%), preservation-reperfusion injury (17.86\%), drug toxicity (14.29\%), and recurrent HCV infection (5.36\%).

Voigtlander et al. [17] reported 496 liver biopsies obtained from 312 liver transplant patients. The main indications of LT were mainly viral hepatitis (28\%), PSC (19\%), and acute liver cell failure (8\%). The main indications for doing liver biopsy were suspected rejection
(66\%) and protocol biopsies (22\%). Other indications were disease recurrence (7\%) and unclear cholestasis (3\%). The average time till liver biopsy was 27 months. The histological findings were acute cellular rejection (36\%), chronic hepatitis (28\%), obstructive cholangiopathy $(7 \%)$, and fatty liver disease $(6 \%)$.

In another report, Fonseca et al. [18] analyzed liver biopsies in Indian liver transplant center. He analyzed the explants, donor liver histopathology, and biopsies done after liver transplantation for pediatric and adult cases. About 58 biopsies were done for 42 patients (adult and

Table 3 Effect of the immunosuppressive drug regimen on the liver histopathological diagnosis

\begin{tabular}{lllll}
\hline Histopathology.1 & \multicolumn{2}{l}{ Immunosuppressive drug regimen } & & \\
\cline { 2 - 5 } & CNI based & mTOR based & Dual CNI and mTOR & Total \\
& $n=18$ & $n=9$ & $1(14.3 \%)$ & $n=34$ \\
\hline Acute rejection & $3(16.7 \%)$ & $3(33.3 \%)$ & $3(42.9 \%)$ & $1(20.6 \%)$ \\
Viral chronic hepatitis & $10(55.6 \%)$ & $4(44.4 \%)$ & $1(14.3 \%)$ & 0.81 \\
Steatohepatitis & $2(11.1 \%)$ & $1(11.1 \%)$ & $0(0 \%)$ & $1(11.8 \%)$ \\
Biliary obstruction & $1(5.6 \%)$ & $0(0 \%)$ & $1(0 \%)$ & $1(2.9 \%)$ \\
CMV infection & $0(0 \%)$ & $1(11.1 \%)$ & $1(14.3 \%)$ & $2(5.9 \%)$ \\
Chronic rejection & $1(5.6 \%)$ & $0(0 \%)$ & $1(14.3 \%)$ & $2(5.9 \%)$
\end{tabular}




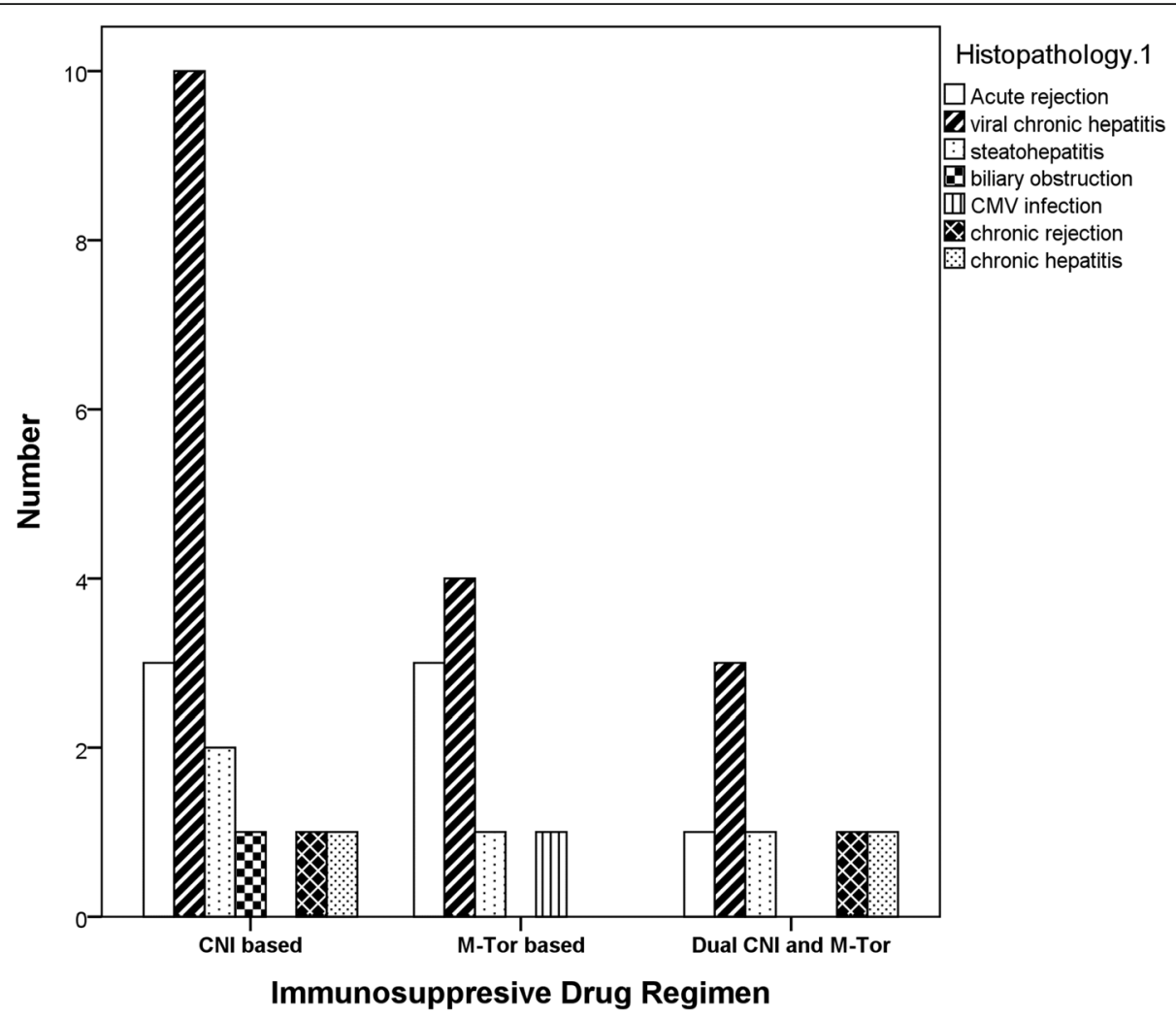

Fig. 4 Impact of the immunosuppressive drug regimen on the histopathology

pediatric). Some patients underwent repeated biopsies. In adults, the most common histopathology was acute cellular rejection.

In the current study, 89 patients were followed up where $38.2 \%$ underwent liver biopsy mainly for suspecting acute rejection or recurrent diseases especially viral ones. Some patients underwent single biopsy and some patients underwent repeated biopsies consistent with other studies [14-16, 18]. The average age was 44 years that is lower than Kanodia et al. [16], and higher than Geramizadeh et al. [15] as he enrolled pediatric cases.

Table 4 Sequential finding of the first, second, and third liver biopsy

\begin{tabular}{llll}
\hline & First biopsy & Second biopsy & Third biopsy \\
\hline Case 1 & Acute rejection & Chronic rejection & \\
Case 2 & Acute rejection & Acute rejection & \\
Case 3 & Biliary obstruction & Chronic rejection & \\
Case 4 & Chronic hepatitis & Chronic hepatitis & Chronic hepatitis \\
Case 5 & Chronic rejection & Viral chronic hepatitis & Acute rejection \\
Case 6 & Viral chronic hepatitis & Viral chronic hepatitis & \\
Case 7 & Viral chronic hepatitis & Viral chronic hepatitis & Chronic rejection \\
Case 8 & Viral chronic hepatitis & Acute rejection & Biliary obstruction \\
Case 9 & Viral chronic hepatitis & Acute rejection & CMV infection \\
\hline
\end{tabular}

Most patients were males in agreement with Kanodia et al. [16]. In comparison to biopsy free patients, most patients had more liver dysfunction that was assessed by the ALBI score

Most of the liver functions are elevated more than 2-3 folds and the wide fluctuations were with AST, ALT, GGT, and ALP. In our center, $91.2 \%$ of cases were positive for HCV. Regarding indications of liver transplantation, the first one was HCV related decompensated liver disease followed by $\mathrm{HCC}$ on top of $\mathrm{HCV}$ related liver disease. In the other studies, the indication was different according to the geographical distribution, so in the Iranian study HBV was the main one [15], whereas alcohol in the Indian study [16].

The average time till the 1st liver biopsy was 19.88 (10-93) months. Some centers did very early liver biopsy within hours postoperatively as with Geramizadeh et al. [15], Kanodia et al. [16], and some center earlier time as with Voigtlander et al. [17]. The different histopathological diagnosis was not affected by the time till biopsy.

The first histopathological diagnosis was recurrent viral hepatitis in $50 \%$ of cases followed by acute cellular rejection, steatohepatitis, and chronic rejection. In fact, the incidence of acute cellular rejection is low (20.6\%) in our study compared to $48.2 \%$ [14], 36\% [17], 47\% [15], and $55.36 \%$ [16]. The most probable explanation that 
some studies applied protocol biopsies [18] and they began earlier postoperative biopsies [15-17]. Acute cellular rejection is very common in the early 3 months post-transplant. Maybe the application of induction therapy with basiliximab in some cases and the triple regimen has decreased the occurrence of acute rejection in our center.

Since three studies did early biopsies, so it was common to report preservation-reperfusion injury $[14,16]$ and hepatic artery thrombosis $[14,15]$.

The incidence of recurrent viral hepatitis especially HCV was high (50\%) compared to 3.7\% [14], 5.36\% [16], and $28 \%$ [17]. The explanation that Egypt is one of the countries of high prevalence of chronic hepatitis C [19].

Regarding liver fibrosis and histological activity, most cases were free of F4 fibrosis where most of them were F1 (38.2\%) and A1 (35.3\%). This was due to regular follow-up and early management of the complications.

None of the patients who underwent biopsy were free of positive diagnostic findings in contrast to Geramizadeh et al. [15], who reported that $17.7 \%$ of the patients had normal histopathology.

On correlation of the indication of liver transplant and the found histopathology, it was found that $50 \%$ of the patients with pre-transplant chronic hepatitis $\mathrm{C}$ had recurrent viral hepatitis.

The varied immunosuppressive drug regimen did not affect that pattern of histopathology. Few patients needed on demand second $(n=9)$ and third biopsy $(n=$ 5). Sometimes the diagnosis was the same as acute rejection and some proceeded to chronic rejection. Patients with viral hepatitis proceeded to other events as rejection in the other biopsies.

The limitations of the study were small number, single centered experienced, and absence of protocol biopsies.

\section{Conclusion}

Liver biopsy is still a useful diagnostic tool in liver transplant recipients.

\begin{abstract}
Abbreviations
AlH: Autoimmune hepatitis; ALBI: Albumin-bilirubin; ALP: Alkaline phosphatase; ALT: Alanine aminotransferase; AST: Aspartate aminotransferase; CMV: Cytomegalovirus; CNI: Calcineurin inhibitors; GGT: Gamma-glutamyl transferase; HBV: Hepatitis B virus; HCC: Hhepatocellular carcinoma; HCV: Hepatitis C virus; INR: International normalized ratio; LT: Liver transplantation; MELD: Model for end-stage liver disease; mTOR: Mechanistic target of rapamycin; PSC: Primary biliary cholangitis; WBC: White blood cell
\end{abstract}

\section{Acknowledgements}

All authors are greatly indebted to members of all departments of National Liver Institute, Menoufia University, Egypt.

\section{Authors' contributions}

EA: initiated the project, designed and implemented the study for application, drafted and revised the paper, AA: analyzed the data, drafted and revised the paper, NE: histopathological examination and interpretation, AE: histopathological examination and interpretation, KA: analyzed the data, one of the surgical team, revised the paper. El: analyzed the data, drafted and revised the paper. ME: analyzed the data, drafted and revised the paper. All authors have read and approved the manuscript.

A poster from this abstract was presented at the Asian Pacific Association for the Study of the Liver 2019.

\section{Funding}

This research and the publication were completely funded by all authors.

\section{Availability of data and materials}

Data used to support the findings of this study are included within the article.

\section{Ethics approval and consent to participate}

The study was conformed to the ethical guidelines of the 1975 Declaration of Helsinki and was approved by the institutional review board of National Liver Institute (NLI IRB 00003413), Menoufia University, Egypt (approval no. 00111/2014). Written informed consents were obtained from both donors and recipients regarding surgery and research.

\section{Consent for publication}

Written informed consents were obtained from both donors and recipients regarding surgery and research. Patient involved in this study agree for publication of data.

\section{Competing interests}

The authors declare that they have no competing interests.

\section{Author details}

${ }^{1}$ Department of Hepatology and Gastroenterology, National Liver Institute, Menoufia University, Shebeen El-Koom 32511, Egypt. 'Department of Pathology, National Liver Institute, Menoufia University, Shebeen El-Koom, Egypt. ${ }^{3}$ Department of Hepatobiliary Surgery, National Liver Institute, Menoufia University, Shebeen El-Koom, Egypt.

Received: 18 December 2019 Accepted: 19 March 2020 Published online: 18 May 2020

\section{References}

1. Gad EH, Alsebaey A, Lotfy M, Eltabbakh M, Sherif AA (2015) Complications and mortality after adult to adult living donor liver transplantation: a retrospective cohort study. Ann Med Surg (Lond) 4:162-171

2. Caiado AH, Blasbalg R, Marcelino AS, da Cunha PM, Chammas MC, da Costa LC et al (2007) Complications of liver transplantation: multimodality imaging approach. Radiographics 27:1401-1417

3. Adeyi O, Fischer SE, Guindi M (2010) Liver allograft pathology: approach to interpretation of needle biopsies with clinicopathological correlation. J Clin Pathol 63:47-74

4. Da J (2006) Liver biopsy interpretation for causes of late liver allograft dysfunction. Hepatology (Baltimore, Md) 44:489-501

5. Abdelsameea E, Alsebaey A, Abdel-Razek W, Ehsan N, Morad W, Salama M et al (2020) Elastography and serum markers of fibrosis versus liver biopsy in 1270 Egyptian patients with hepatitis C. Eur J Gastroenterol Hepatol

6. C. RD, H. CS, D. GZ, C. NR, D. SA. Liver biopsy. Hepatology (Baltimore, Md) 2009:49:1017-1044.

7. Hiraoka A, Kumada T, Kudo M, Hirooka M, Tsuji K, Itobayashi E et al (2017) Albumin-Bilirubin (ALBI) grade as part of the evidence-based clinical practice guideline for HCC of the Japan Society of Hepatology: a comparison with the liver damage and child-pugh classifications. Liver Cancer 6:204-215

8. De Simone P, Fagiuoli S, Cescon M, De Carlis L, Tisone G, Volpes R et al (2017) Use of everolimus in liver transplantation: recommendations from a working group. Transplantation 101:239-251

9. Cholongitas E, Goulis I, Theocharidou E, Antoniadis N, Fouzas I, Giakoustidis $D$ et al (2014) Everolimus-based immunosuppression in liver transplant recipients: a single-centre experience. Hepatol Int 8:137-145

10. Intraobserver and interobserver variations in liver biopsy interpretation in patients with chronic hepatitis C. The French METAVIR Cooperative Study Group. Hepatology (Baltimore, Md) 1994;20:15-20.

11. Johnson PJ, Berhane S, Kagebayashi C, Satomura S, Teng M, Reeves HL et al (2015) Assessment of liver function in patients with hepatocellular 
carcinoma: a new evidence-based approach—-the ALBI grade. J Clin Onco 33:550-558

12. P. WJ, M. HA, W. BS. MELD score, allocation, and distribution in the United States. Clin Liver Dis 2013;2:148-151.

13. Rockey DC, Caldwell SH, Goodman ZD, Nelson RC, Smith AD (2009) Liver biopsy. Hepatology (Baltimore, Md) 49:1017-1044

14. Yu YY, Ji J, Zhou GW, Shen BY, Chen H, Yan JQ et al (2004) Liver biopsy in evaluation of complications following liver transplantation. World J Gastroenterol 10:1678-1681

15. Geramizadeh B, Motevalli D, Nikeghbalian S, Malek Hosseini SA (2013) Histopathology of post-transplant liver biopsies, the first report from iran. Hepatitis Monthly 13:e9389

16. Kanodia KV, Vanikar AV, Modi PR, Patel RD, Suthar KS, Nigam LK et al (2015) Histological and clinicopathological evaluation of liver allograft biopsy: an initial experience of fifty six biopsies. J Clin Diagn Res 9:EC17-EC20

17. Voigtlander T, Alten TA, Kirstein MM, Lehner F, Manns MP, Schlue J et al (2017) Clinical impact of liver biopsies in liver transplant recipients. Ann Transplant 22:108-114

18. Fonseca F, Kulkarni B, Hastak M, Kumaran V, Varma V, Kapoor S (2018) An overview of liver transplant pathology: data from a tertiary referral centre in Western India. Ann Hepatol 17:426-436

19. Abozeid M, Alsebaey A, Abdelsameea E, Othman W, Elhelbawy M, Rgab A et al (2018 Oct) High efficacy of generic and brand direct acting antivirals in treatment of chronic hepatitis C. Int J Infect Dis. 75:109-114. https://doi.org/ 10.1016/j.jijd.2018.07.025

\section{Publisher's Note}

Springer Nature remains neutral with regard to jurisdictional claims in published maps and institutional affiliations.

\section{Submit your manuscript to a SpringerOpen ${ }^{\circ}$ journal and benefit from:}

- Convenient online submission

- Rigorous peer review

- Open access: articles freely available online

- High visibility within the field

- Retaining the copyright to your article

Submit your next manuscript at $\boldsymbol{\nabla}$ springeropen.com 